\title{
Multi-scale simulations of metamagnetic martensite transition in
}

\begin{abstract}
NiCoMnIn
H. B. Huang ${ }^{1 * \#}$, W. Q. He ${ }^{1 *}$, G. P. Cao ${ }^{1 *}$, J. J. Wang ${ }^{1}$, Z. H. Liu ${ }^{1}$, X. Q. Ma ${ }^{1 \#}$

${ }^{1}$ Department of Physics, University of Science and Technology Beijing, Beijing, 100083, China

ABSTRACT

A multi-scale theoretical model is performed to investigate magnetic field-induced metamagnetic martensite transition in NiCoMnIn alloys. First, the total energy is calculated to demonstrate antiferromagnetic-ferromagnetic transition from first principle calculation. Second, the transition temperature from antiferromagnetic to ferromagnetic can be tuned by the external magnetic field and hydrostatic pressure in thermodynamic calculation, which is consistent with experimental results. Third, phase field simulation found that martensite variant boundaries are superposed with the $90^{\circ}$ antiferromagnetic magnetic domain walls, revealing magneto-structural coupling between structural and magnetic domain structures. It is demonstrated that this multiscale model is very effective for describing antiferromagnetic-ferromagnetic accompanied with martensite-austenite transitions with magnetostructural coupling.
\end{abstract}

\section{KEYWORDS}

Phase field simulations, Metamagnetic transition, NiCoMnIn, Magnetostructural coupling

\#Corresponding author. E-mail: hbhuang@ustb.edu.cn (H. B. Huang), xqma@ sas.ustb.edu.cn (X. Q. Ma)

*Those authors contributed equally to this work. 


\section{Introduction}

Heusler alloys NiMnIn have attracted great attention due to their magnetic shape memory and magnetocaloric effects in the past decades ${ }^{1}$. In those alloys, the ferroelastic and ferromagnetic orderings are coupled, thus a magnetic field can manipulate the reorientation of ferroelastic domains to obtain giant strain. The strain is one order of magnitude higher than that of the giant magnetostrictive materials ${ }^{2}$. On the other hand, the large entropy change, which is an essential property of magnetic refrigeration material ${ }^{3}$, can be realized under the driving of an external magnetic field.

The key to finding metamagnetic materials is in search for the materials whose phase transition temperature is sensitive to the external magnetic field. The experiments showed that doping Co in NiMnX alloys (X=Ga, In, Sn, Sb) can improve the response sensitivity of the phase transition temperature ${ }^{4}$. For NiCoMnIn alloy, there are two-phase transitions with the decrease of temperature, from paramagnetic (PM) austenite (Aust) to ferromagnetic (FM) Aust, then to antiferromagnetic (AFM) martensite (Mart). In addition, the phase transition_temperatures can be adjusted by the external magnetic field or stress. Consequently, several similar alloys such as $\mathrm{NiCoMnSn}{ }^{5}, \mathrm{NiCoMnSb}{ }^{6}, \mathrm{NiCoMnGa}^{7}$, and even their $\mathrm{Cu}$-coped counterparts ${ }^{8-10}$ were investigated for the magnetocaloric effect. In addition, Landau phenomenological theory ${ }^{11,12}$ and the first principle calculation ${ }^{13-15}$ were developed to describe Mart phase transitions. Although several groups have already simulated FM in Mart phase transitions of $\mathrm{NiMnGa}{ }^{16-20}$, they focused on the phase stability and domain orientation without considering the metamagnetic behavior. There is no existing theoretical description of systems with metamagnetic features ${ }^{21}$.

We investigate the magnetic phase transition as a function of temperature, and the saturation magnetization $M_{s}$ of NiCoMnIn is temperature-dependent. Therefore, we propose to use the 
time-dependent Ginzburg-Landau (TDGL) equation to describe the metamagnetic transition ${ }^{22,23}$. In this paper, we firstly calculated the total energy as a function of the magnetic unit to indentify the metamagnetic transition temperature by using first principle calculation. Then the metamagnetic transition temperature can be tuned by the external magnetic field and hydrostatic pressure according to the thermodynamic calculations. Furthermore, the phase field simulation is performed to investigate the microstructure of magnetic domain.

\section{Theoretical Model}

In this work, we performed the calculation based on the density functional theory (DFT) using the Vienna ab initio simulation package (VASP) ${ }^{24,25}$. The electronic exchange-correlation were described within a spin polarized generalized-gradient approximation (GGA) using the functional proposed by Perdew et $\mathrm{al}^{26}$. The electron-ion interaction was described with the projector augmented-wave (PAW) formalism ${ }^{27}$. We use $\mathrm{L} 2{ }_{1}$ cubic structure (austenite) which is the conventional 16-atoms cell $\left(\mathrm{Ni}_{8} \mathrm{Mn}_{6} \mathrm{In}_{2}\right)$ substituting two In atoms with $\mathrm{Mn}$ atoms and $\mathrm{L}_{0}$ tetragonal structure (martensite) with $\mathrm{c} / \mathrm{a}=1.27$ for the calculation. The AFM configuration is that the magnetic moment of Mn atoms at In sites is antiparallel to that at the original sites. Cut-off energy was truncated at $450 \mathrm{eV}$ for the plane wave basis, and a Monkhorst-Pack ${ }^{28}$ type $11 \times 11 \times$ 11 k-point grid was adopted for energy calculation. In the fixed spin moment $(\mathrm{FSM})^{29,} 30$ procedure, we determine the total energy $E$ of a system constrained to have a given magnetic moment $M$ per unit cell.

For the thermodynamic calculation, the directions of spins do not change significantly from one lattice point to another in the FM materials. However, spins of an AFM phase usually have opposite directions from lattice point to lattice point. As shown in Figure 1, if the AFM is divided 
into sublattices ${ }^{31}$, the magnetic moments can be described by two antiparallel FM sublattices of $\mathrm{A}$ and $\mathrm{B}$ whose magnetizations are expressed by $\mathbf{M}_{1}$ and $\mathbf{M}_{2}$. Furthermore, we define $\mathbf{M}=\left(\mathbf{M}_{1}+\mathbf{M}_{2}\right) / 2 M_{s}$ and $\mathbf{L}=\left(\mathbf{M}_{1}-\mathbf{M}_{2}\right) / 2 M_{s}$ as FM and AFM order parameters, respectively, and $M_{\mathrm{s}}$ indicates the constant magnetization at $280 \mathrm{~K}$ and zero external magnetic field. If $\mathbf{M}_{1}$ and $\mathbf{M}_{2}$ are parallel, $\mathbf{M} \neq 0$, and $\mathbf{L}=0$. Otherwise, if $\mathbf{M}_{1}$ and $\mathbf{M}_{2}$ are antiparallel, $\mathbf{M}=0$, and $\mathbf{L} \neq 0$.

It is observed in experiments that NiCoMnIn single crystal stabilizes in three phases, i.e., PM Aust phase above 382K, FM Aust phase between 285K and 380K, AFM Mart phase below 280K. The transition temperatures from PM to FM (Curie temperature $T_{C}$ ), from FM to AFM $\left(T_{A}\right)$, and from Aust to Mart $\left(T_{M}\right)$ can be shifted by applying external stimulus such as magnetic field or stress. Under the external magnetic field or stress, the magnetic domain and Mart variants will evolve to minimize the total free energy.

For phase field model, we focus on the metamagnetic transitions, and the magnetization $\mathbf{M}$ of NiCoMnIn is temperature dependent. Therefore, we use three TDGL equations to evolve the FM magnetization $\mathbf{M}, \mathrm{AFM}$ magnetization $\mathbf{L}$ and Mart strain $\varepsilon_{i i}^{0}$ :

$$
\frac{\partial \mathbf{M}(\mathbf{r}, t)}{\partial t}=-L^{m} \frac{\delta E}{\delta \mathbf{M}}, \frac{\partial \mathbf{L}(\mathbf{r}, t)}{\partial t}=-L^{l} \frac{\delta E}{\delta \mathbf{L}}, \frac{\partial \varepsilon_{i i}^{0}(\mathbf{r}, t)}{\partial t}=-L^{e} \frac{\delta E}{\delta \varepsilon_{i i}^{0}},
$$

where $L^{m}, L^{l}$ and $L^{e}$ are kinetic coefficients relating to the FM, AFM domain evolution and the twin boundary mobility.

The total free energy for NiCoMnIn in the thermodynamic calculation can be expressed by ${ }^{21}$, 


$$
\begin{aligned}
E=\int\{ & \alpha_{1}\left(M_{x}^{2}+M_{y}^{2}+M_{z}^{2}\right)+\alpha_{11}\left(M_{x}^{4}+M_{y}^{4}+M_{z}^{4}\right)+\alpha_{111}\left(M_{x}^{6}+M_{y}^{6}+M_{z}^{6}\right) \\
& +\alpha_{12}\left(M_{x}^{2} M_{y}^{2}+M_{x}^{2} M_{z}^{2}+M_{y}^{2} M_{z}^{2}\right)+\alpha_{123} M_{x}^{2} M_{y}^{2} M_{z}^{2} \\
& +\alpha_{112}\left[M_{x}^{2}\left(M_{y}^{2}+M_{z}^{2}\right)+M_{y}^{2}\left(M_{x}^{2}+M_{z}^{2}\right)+M_{z}^{2}\left(M_{x}^{2}+M_{y}^{2}\right)\right] \\
& +\beta_{1}\left(L_{x}^{2}+L_{y}^{2}+L_{z}^{2}\right)+\beta_{11}\left(L_{x}^{4}+L_{y}^{4}+L_{z}^{4}\right)+\beta_{111}\left(L_{x}^{6}+L_{y}^{6}+L_{z}^{6}\right) \\
& +\beta_{12}\left(L_{x}^{2} L_{y}^{2}+L_{x}^{2} L_{z}^{2}+L_{y}^{2} L_{z}^{2}\right)+\beta_{123} L_{x}^{2} L_{y}^{2} L_{z}^{2} \\
& +\beta_{112}\left[L_{x}^{2}\left(L_{y}^{2}+L_{z}^{2}\right)+L_{y}^{2}\left(L_{x}^{2}+L_{z}^{2}\right)+L_{z}^{2}\left(L_{x}^{2}+L_{y}^{2}\right)\right] \\
& +\mu_{11}\left(M_{x} L_{x}+M_{y} L_{y}+M_{z} L_{z}\right)^{2}+\mu_{12}\left(M_{x}^{2}+M_{y}^{2}+M_{z}^{2}\right)\left(L_{x}^{2}+L_{y}^{2}+L_{z}^{2}\right) \\
& -\mathbf{H}_{\mathrm{d}} \cdot \mathbf{M}^{2}+Q_{1} e_{1}^{2}+Q_{2}\left(e_{2}^{2}+e_{3}^{2}\right)+Q_{3}\left(e_{4}^{2}+e_{5}^{2}+e_{6}^{2}\right) \\
& +Q_{4} e_{1}\left(e_{2}^{2}+e_{3}^{2}\right)+Q_{5} e_{3}\left(e_{3}^{2}-3 e_{2}^{2}\right)+Q_{6}\left(e_{2}^{2}+e_{3}^{2}\right)^{2}+\frac{1}{2} c_{i j k l}\left(\varepsilon_{i j}-\varepsilon_{i j}^{0}\right)\left(\varepsilon_{k l}-\varepsilon_{k l}^{0}\right) \\
& +\gamma_{0} e_{1}\left(M_{x}^{2}+M_{y}^{2}+M_{z}^{2}\right)+\gamma_{1}\left[e_{2}\left(M_{x}^{2}-M_{y}^{2}\right) / 2+e_{3}\left(3 M_{z}^{2}-\mathbf{M}^{2}\right) / \sqrt{6}\right] \\
& +\gamma_{2}\left(e_{4} M_{x} M_{y}+e_{5} M_{y} M_{z}+e_{6} M_{x} M_{z}\right) \\
& +\lambda_{0} e_{1}\left(L_{x}^{2}+L_{y}^{2}+L_{z}^{2}\right)+\lambda_{1}\left[e_{2}\left(L_{x}^{2}-L_{y}^{2}\right) / 2+e_{3}\left(3 L_{z}^{2}-\mathbf{L}^{2}\right) / \sqrt{6}\right] \\
& \left.+\lambda_{2}\left(e_{4} L_{x} L_{y}+e_{5} L_{y} L_{z}+e_{6} L_{x} L_{z}\right)-\left(H_{x} M_{x}+H_{y} M_{y}+H_{z} M_{z}\right)-\sigma e_{1}\right\} d V
\end{aligned}
$$

where $\alpha_{1}, \alpha_{11}, \alpha_{111}, \alpha_{12}, \alpha_{123}, \alpha_{112}, \beta_{1}, \beta_{11}, \beta_{111}, \beta_{12}, \beta_{123}, \beta_{112}, \mu_{11}, \mu_{12}$ are exchange-interaction coefficients, $\mathbf{H}_{\mathrm{d}}$ is the demagnetization field that arises from the long-range interactions among the magnetic moments in the system. The linear combination of the deformation tensor components $e_{i}$ can be defined in terms of the transformation strains, $e_{1}=\left(\varepsilon_{11}^{0}+\varepsilon_{22}^{0}+\varepsilon_{33}^{0}\right) / \sqrt{3}$, $e_{2}=\left(\varepsilon_{11}^{0}-\varepsilon_{22}^{0}\right) / \sqrt{2}, \quad e_{3}=\left(2 \varepsilon_{33}^{0}-\varepsilon_{11}^{0}-\varepsilon_{22}^{0}\right) / \sqrt{6}, \quad e_{4}=\varepsilon_{23}^{0}, \quad e_{5}=\varepsilon_{13}^{0}, \quad e_{6}=\varepsilon_{12}^{0}$. Additionally, $Q_{1}, Q_{2}, Q_{3}$, $Q_{4}, Q_{5}, Q_{6}$ are the linear combinations of the second-, third-, and fourth-order elastic moduli, respectively; $Q_{1}=\left(C_{11}+2 C_{12}\right) / 2 \sqrt{3}, \quad Q_{2}=\left(C_{11}-C_{12}\right) / 2, Q_{3}=C_{44}, \quad Q_{4}=\left(C_{111}-C_{123}\right) / 2 \sqrt{3}$, $Q_{5}=\left(C_{111}-3 C_{112}+2 C_{123}\right) / 18 \sqrt{6}, Q_{6}=\left(C_{1111}+C_{1112}-3 C_{1122}-8 C_{1123}\right) / 192 . \varepsilon_{i j}$ is the total strain, and $\varepsilon_{i j}^{0}$ is the stress-free eigenstrain. $c_{i j k l}$ represents the components of the second-order elastic stiffness tensor, and $\mathrm{C}_{\mathrm{ij}}$ are the elastic constants in Voigt notation, i.e., $C_{11}=c_{1111}, C_{12}=c_{1122}$, 
$C_{44}=c_{2323} \cdot \gamma_{0}, \lambda_{0}$ are the volume magnetostriction constants, and $\gamma_{1,2}, \lambda_{1,2}$ are the anisotropic magnetostriction constants of the FM and AFM phases, $\mathbf{H}$ and $\sigma$ are the external magnetic field and stress. In addition, the total energy in the phase-field simulations also includes the gradient and exchange energy densities which can be calculated by,

$$
\begin{aligned}
& f_{\text {grad }}=g\left[\left(\varepsilon_{11,1}^{0}\right)^{2}+\left(\varepsilon_{11,2}^{0}\right)^{2}+\left(\varepsilon_{11,3}^{0}\right)^{2}+\left(\varepsilon_{22,1}^{0}\right)^{2}+\left(\varepsilon_{22,2}^{0}\right)^{2}+\left(\varepsilon_{22,3}^{0}\right)^{2}+\left(\varepsilon_{33,1}^{0}\right)^{2}+\left(\varepsilon_{33,2}^{0}\right)^{2}+\left(\varepsilon_{33,3}^{0}\right)^{2}\right] \\
& f_{\text {exch }}=A_{M}\left[\left(\nabla M_{x}\right)^{2}+\left(\nabla M_{y}\right)^{2}+\left(\nabla M_{z}\right)^{2}\right]+A_{L}\left[\left(\nabla L_{x}\right)^{2}+\left(\nabla L_{y}\right)^{2}+\left(\nabla L_{z}\right)^{2}\right]
\end{aligned}
$$

where $g$ is the isotropic gradient energy coefficient, and $A_{M}$ and $A_{L}$ are the exchange constants of FM and AFM. The model size of $64 \times 64 \times 1$ is employed. The coefficients are obtained from the thermodynamic simulations ${ }^{22}$ and can be found in our previous paper ${ }^{23}$.

\section{Results and Discussions}

As shown in Figure 2, we use first principle calculation to obtain the total energy as a function of the total magnetic moment of the unit cell. Our results show that there is a tetragonal phase (Mart) at the small magnetic moment $\left(7.98 \mathrm{u}_{\mathrm{B}}\right)$, and a cubic phase (Aust) at the large magnetic moment $\left(25.6 \mathrm{u}_{\mathrm{B}}\right)$. According to the curve, the total energy of the tetragonal phase with small magnetic moment is lower than that of cubic phase with high magnetic moment. It means that the tetragonal structure (Mart) is more stable than the cubic phase (Aust) at zero Kelvin.

Using the fitted coefficients, we calculated the metamagnetic transition of NiCoMnIn alloy single crystalline from Eq. (2) by minimizing the total free energy from thermodynamic calculation. Figure 3 shows the total free energy as a function of temperature with different external magnetic fields. For the black line, the free energy of AFM Mart increases with the temperature and becomes zero at around 300K. The free energy of FM Aust under different external magnetic fields also increases with the temperature. The total free energy for AFM Mart 
and FM Aust show independent and dependent on the magnetic field, respectively. The external magnetic field will reduce the total free energy of FM at the same temperature. The intersections of AFM and FM free energies curves are the transition temperatures from AFM Mart to FM Aust. If the temperature is lower than that of the intersection, the material is AFM Mart. Otherwise, the material is FM Aust. We observe that the transition temperature $\left(T_{A}\right.$ or $\left.T_{M}\right)$ is decreasing with the increase of external magnetic fields.

Under a hydrostatic pressure, the applied stress tensors satisfy $\sigma=-p$, where $p$ is the hydrostatic pressure. As shown in Figure 4, the AFM-FM transition temperature as a function of magnetic field and hydrostatic pressure were calculated from thermodynamic simulation. Figure 4 (a) shows the AFM-FM transition temperature decrease with the increase of magnetic field, and FM-PM transition temperature is increasing with the magnetic field, which are consistent with the experimental data. The external magnetic field will favor FM state, which results to the decrease of AFM-FM and the FM-PM transition temperatures. As shown in Figure 4(b), both AFM-FM transition temperature is increasing with the hydrostatic pressure, however FM-PM transition temperature is independent on the hydrostatic pressure.

As shown in Figures 5 (a) and (b), we calculated the FM, AFM magnetizations $\mathbf{M}, \mathbf{L}$ and Mart strain $\varepsilon_{i i}^{0}$ at the temperature of $200 \mathrm{~K}$ from the initial random distribution from phase-field simulations. We observed that the FM magnetization $\mathbf{M}$ is zero, and the AFM magnetization $\mathbf{L}$ and Mart strain are increasing with the evolution. It indicates that the metamagnetic transition can be described by our model. Figures 5 (c), (d) and (e) show FM domains, AFM domains and Mart variants at the low temperature. The arrows of figure 5 (d) represents the directions of $\mathbf{L}$ which is along $-\mathrm{x},+\mathrm{x},-\mathrm{y}$ and $+\mathrm{y}$ axes. In figure 5 (e), yellow, and purple areas represent Mart variant $\mathrm{A}$, and Mart variant $\mathrm{B}$, respectively. We clearly observed the AFM domains coupled to 
the Mart variants at low temperatures. The weak magnetic domain can be described at the low temperature of Mart phase in which the Mart variant boundaries are superposed with the $90^{\circ}$ magnetic domain wall as an evidence of magnetostructural coupling. The magnetic domains and martensite variants are coupled together due to the magnetoelastic energy. The magnetoelastic energy density can be calculated by,

$$
\begin{aligned}
f_{\text {coup }}= & \gamma_{0} e_{1}\left(M_{x}^{2}+M_{y}^{2}+M_{z}^{2}\right)+\gamma_{1}\left[e_{2}\left(M_{x}^{2}-M_{y}^{2}\right) / 2+e_{3}\left(3 M_{z}^{2}-\mathbf{M}^{2}\right) / \sqrt{6}\right] \\
& +\lambda_{0} e_{1}\left(L_{x}^{2}+L_{y}^{2}+L_{z}^{2}\right)+\lambda_{1}\left[e_{2}\left(L_{x}^{2}-L_{y}^{2}\right) / 2+e_{3}\left(3 L_{z}^{2}-\mathbf{L}^{2}\right) / \sqrt{6}\right]
\end{aligned}
$$

It is noticed that the magnetoelastic energy is proportional to two times order of $M_{i}$ and $L_{i}(i=x, y$, and $z$ ). For the $180^{\circ}$ domain wall, $M_{i}$ or $-M_{i}\left(L_{i}\right.$ or $\left.-L_{i}\right)$ will not change the magnetoelastic energy. However, $M_{i}$ or $-M_{i}\left(L_{i}\right.$ or $\left.-L_{i}\right)$ will change in the $90^{\circ}$ domain wall. Therefore, the martensite variant boundaries are coupled with $90^{\circ}$ domain walls. Furthermore, FM domain almost equals to zero at the low temperature. However, we can observe the maximum at the $90^{\circ}$ domain wall which indicates the metamagnetic transition will happen in this area.

As shown in Figures 6 (a) and (b), we calculated the magnetization $\mathbf{M}$ as a function of external magnetic field $\mathbf{H}$ with different temperatures from phase-field simulations. The curves in Figure 6 (a) at 190 and $320 \mathrm{~K}$ exhibit typical AFM and FM M-H behavior, respectively. The curves with the temperature from 250 to $290 \mathrm{~K}$ exhibit metamagnetic behavior. The applied magnetic field induces a reversal transformation from AFM to FM. During transformation, a mixed structure containing AFM and FM phases exists at 250 290 K. The hysteresis loops display sigmoid shapes, in which the magnetization rapidly increases and then slowly increases with increasing applied magnetic field strength. The magnetization does not saturate, even at 10 $\mathrm{T}$ [5]. If the temperature continues to increase, the magnetic transition from FM to PM phase would occur at $T_{C}$. Figure 6 (b) shows that the magnetization is low when the temperature 
increases to $490 \mathrm{~K}$, and that the $M(H)$ curves are linear at each temperature. Therefore, there are three types of magnetic field-induced magnetization curves, and these exhibit $M(H)$ typical behaviors of AFM, FM and PM, as a function of temperature. From the magnetization curves, we observe the magnetic field-induced metamagnetic transition, which is coupled to the Mart structural transformation.

\section{Conclusions}

We developed a multi-scale model to study the metamagnetic transition and domain structure. The metamagnetic transition from AFM to FM was obtained from DFT calculation. The transition temperature from AFM to FM can be tuned by the external magnetic field and hydrostatic pressure in thermodynamic calculation, which is consistent with the experimental results. It is found that the Mart variant boundaries are superposed with $90^{\circ}$ AFM magnetic domain walls, revealing magneto-structural coupling between the structural and the magnetic domain structures. Thus, this method can be effectively used to describe the magnetic field induced metamagnetic transition, and explain the mechanism of magnetostructural coupling as well as facilitate applications in a Mart transition system.

\section{Acknowledgements}

This work was sponsored by the National Science Foundation of China (11174030) and (11504020).

\section{References}

1. K. Ullakko, J. Huang, C. Kantner, R. O’handley and V. Kokorin, Appl Phys Lett 69, 1966 (1996).

2. A. Sozinov, A. A. Likhachev, N. Lanska and K. Ullakko, Appl Phys Lett 80 (10), 1746-1748 (2002).

3. V. K. Pecharsky and J. K. A. Gschneidner, Phys Rev Lett 78 (23), 4494-4497 (1997).

4. R. Kainuma, Y. Imano, W. Ito, Y. Sutou, H. Morito, S. Okamoto, O. Kitakami, K. Oikawa, A. Fujita, T. Kanomata and K. Ishida, Nature 439 (7079), 957-960 (2006). 
5. K. Ito, W. Ito, R. Y. Umetsu, M. Nagasako, R. Kainuma, A. Fujita, K. Oikawa and K. Ishida, Materials Transactions 49 (8), 1915 (2008).

6. S. Y. Yu, L. Ma, G. D. Liu, Z. H. Liu, J. L. Chen, Z. X. Cao, G. H. Wu, B. Zhang and X. X. Zhang, Applied Physics Letters 90 (24), 242501 (2007).

7. S. Y. Yu, Z. X. Cao, L. Ma, G. D. Liu, J. L. Chen, G. H. Wu, B. Zhang and X. X. Zhang, Applied Physics Letters 91 (10), 102507 (2007).

8. C. B. Jiang, J. M. Wang, P. P. Li, A. Jia and H. B. Xu, Applied Physics Letters 95 (1), 012501 (2009).

9. B. Gao, J. Shen, F. X. Hu, J. Wang, J. R. Sun and B. G. Shen, Applied Physics a-Materials Science \& Processing 97 (2), 443 (2009).

10. V. K. Sharma, M. K. Chattopadhyay, A. Khandelwal and S. B. Roy, Physical Review B 82 (17), 172411 (2010).

11. E. Hoffmann, H. Herper, P. Entel, S. G. Mishra, P. Mohn and K. Schwarz, Phys Rev B 47 (10), $5589-5596$ (1993).

12. F. Falk, Acta Metallurgica 28 (12), 1773-1780 (1980).

13. E. Şaşığlu, L. Sandratskii and P. Bruno, Phys Rev B 70 (2), 024427 (2004).

14. Y. Kurtulus, R. Dronskowski, G. D. Samolyuk and V. P. Antropov, Phys Rev B 71 (1), 014425 (2005).

15. M. A. Uijttewaal, T. Hickel, J. Neugebauer, M. E. Gruner and P. Entel, Phys Rev Lett 102 (3), 035702 (2009).

16. J. X. Zhang and L. Q. Chen, Philosophical Magazine Letters 85 (10), 533-541 (2005).

17. Y. M. Jin, Acta Mater 57 (8), 2488-2495 (2009).

18. P. P. Wu, X. Q. Ma, J. X. Zhang and L. Q. Chen, J Appl Phys 104 (7), 073906 (2008).

19. P. P. Wu, X. Q. Ma, J. X. Zhang and L. Q. Chen, Philosophical Magazine 91 (16), 2102-2116 (2011).

20. L. J. Li, J. Y. Li, Y. C. Shu, H. Z. Chen and J. H. Yen, Appl Phys Lett 92 (17), 172504 (2008).

21. V. Buchel'nikov, S. Taskaev, M. Zagrebin and P. Entel, Jetp Lett+ 85 (11), 560-564 (2007).

22. J. Wang, X. Ma, H. Huang, W. He, Z. Liu and L.-Q. Chen, Journal of Applied Physics 114 (1), 013504 (2013).

23. H. B. Huang, X. Q. Ma, J. J. Wang, Z. H. Liu, W. Q. He and L. Q. Chen, Acta Mater 83 (0), $333-340$ (2015).

24. G. Kresse and J. Hafner, Phys Rev B 47 (1), 558-561 (1993).

25. G. F. Kresse, Phys Rev B 54 (16), 11169-11186 (1996).

26. K. B. J. P. Perdew, M. Ernzerhof, Phys Rev Lett 77 (38) (1996).

27. P. E. Blöchl, Phys Rev B 50 (24), 17953-17979 (1994).

28. M. Methfessel and A. Paxton, Phys Rev B 40 (6), 3616-3621 (1989).

29. V. L. Moruzzi, P. M. Marcus, K. Schwarz and P. Mohn, Phys Rev B 34 (3), 1784-1791 (1986).

30. M. Uijttewaal, T. Hickel, J. Neugebauer, M. Gruner and P. Entel, Phys Rev Lett 102 (3) (2009).

31. D. Suess, T. Schrefl, W. Scholz, J.-V. Kim, R. Stamps and J. Fidler, Magnetics, IEEE Transactions on 38 (5), 2397-2399 (2002).

\section{Figure Captions}

Figure 1 (a) Schematic diagram of an AFM divided into two sublattices, and (b) schematic diagrams of the magnetic and structure transitions with the decrease of the temperature.

Figure 2 The total energy as a function of total magnetic moment of the unit cell from DFT calculation.

Figure 3 The total free energy as a function of temperature with different external magnetic fields from thermodynamic calculation.

Figure 4 The AFM-FM transition temperatures as a function of magnetic field and hystrostatic 
pressure from thermodynamic calculation.

Figure 5 (a) (b) FM and AFM magnetizations and Mart strain average magnitude evolution (c) (e) FM, AFM domains and Mart variants spatial distribution evolution from phase-field simulation.

Figure 6 (a) (b) Magnetization loops at different temperatures from phase-field simulation. 
(a) Antiferromagnetics
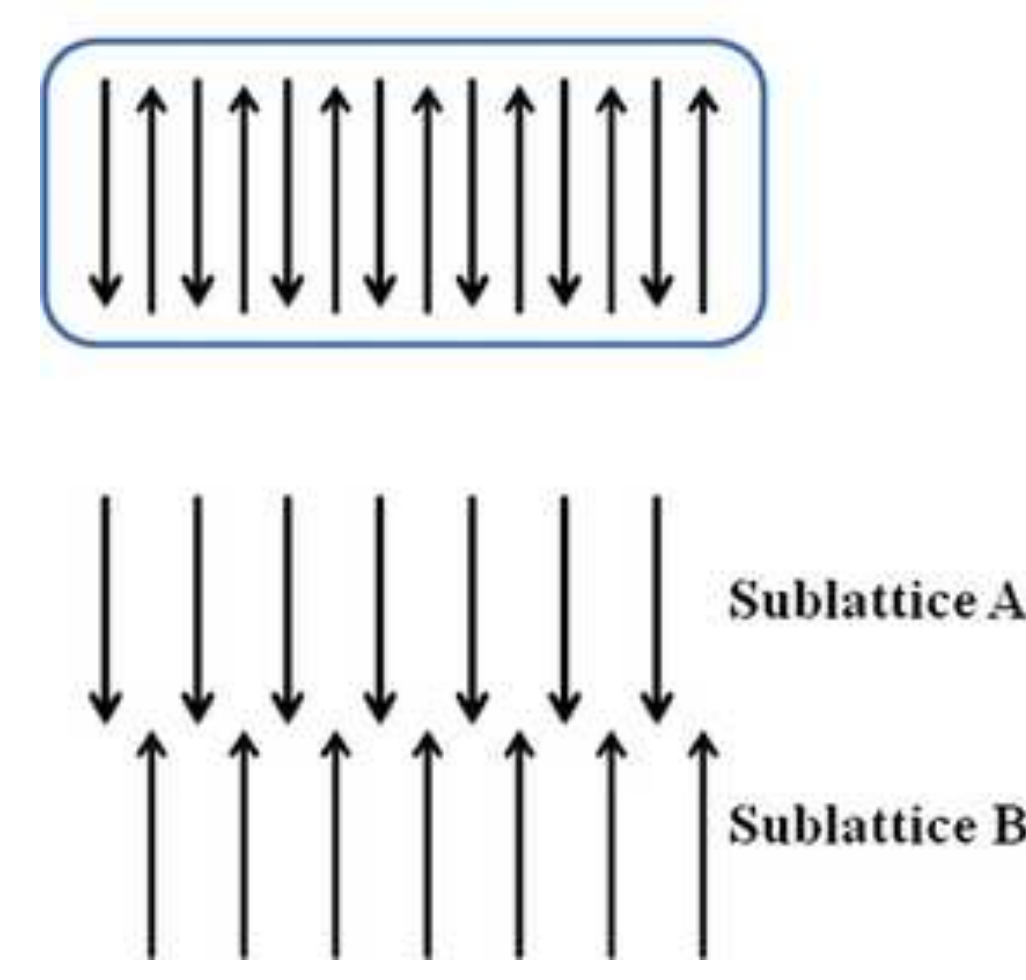

(b)

Temperature

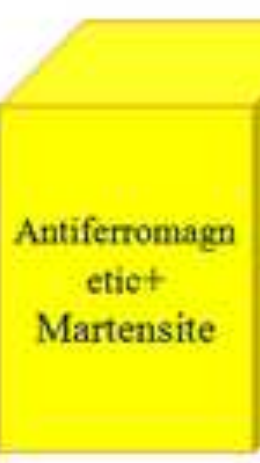

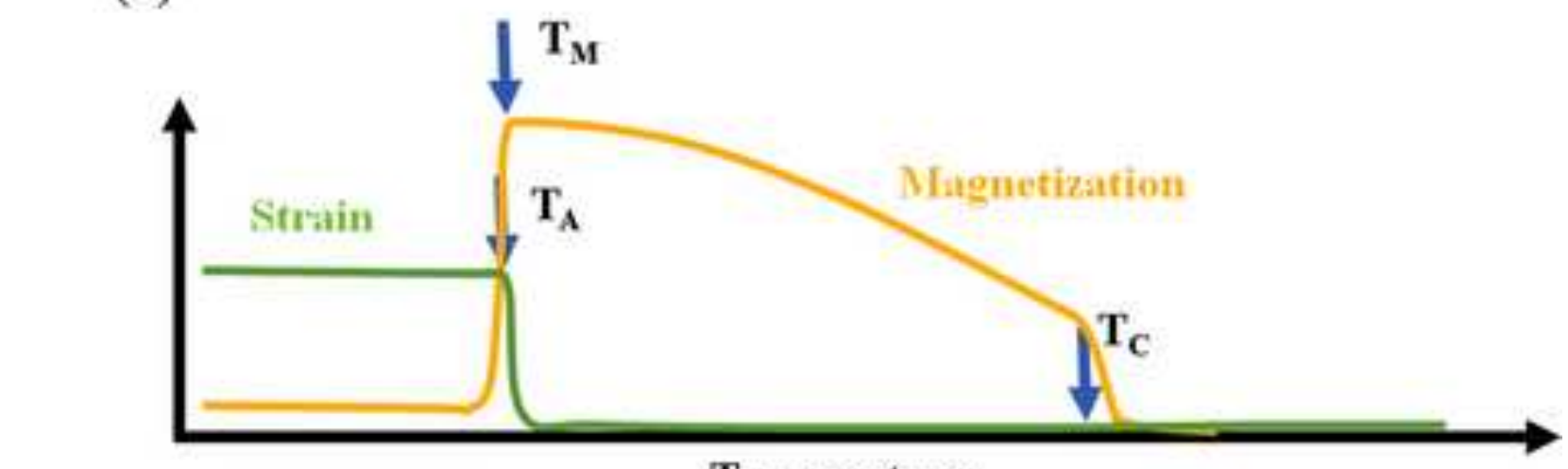

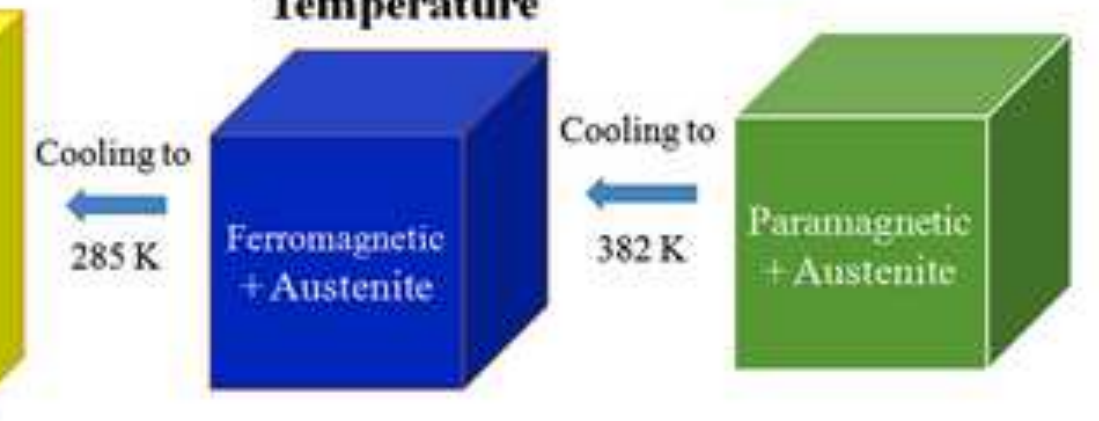

$$
\text { . }
$$

$$
\text { . }
$$




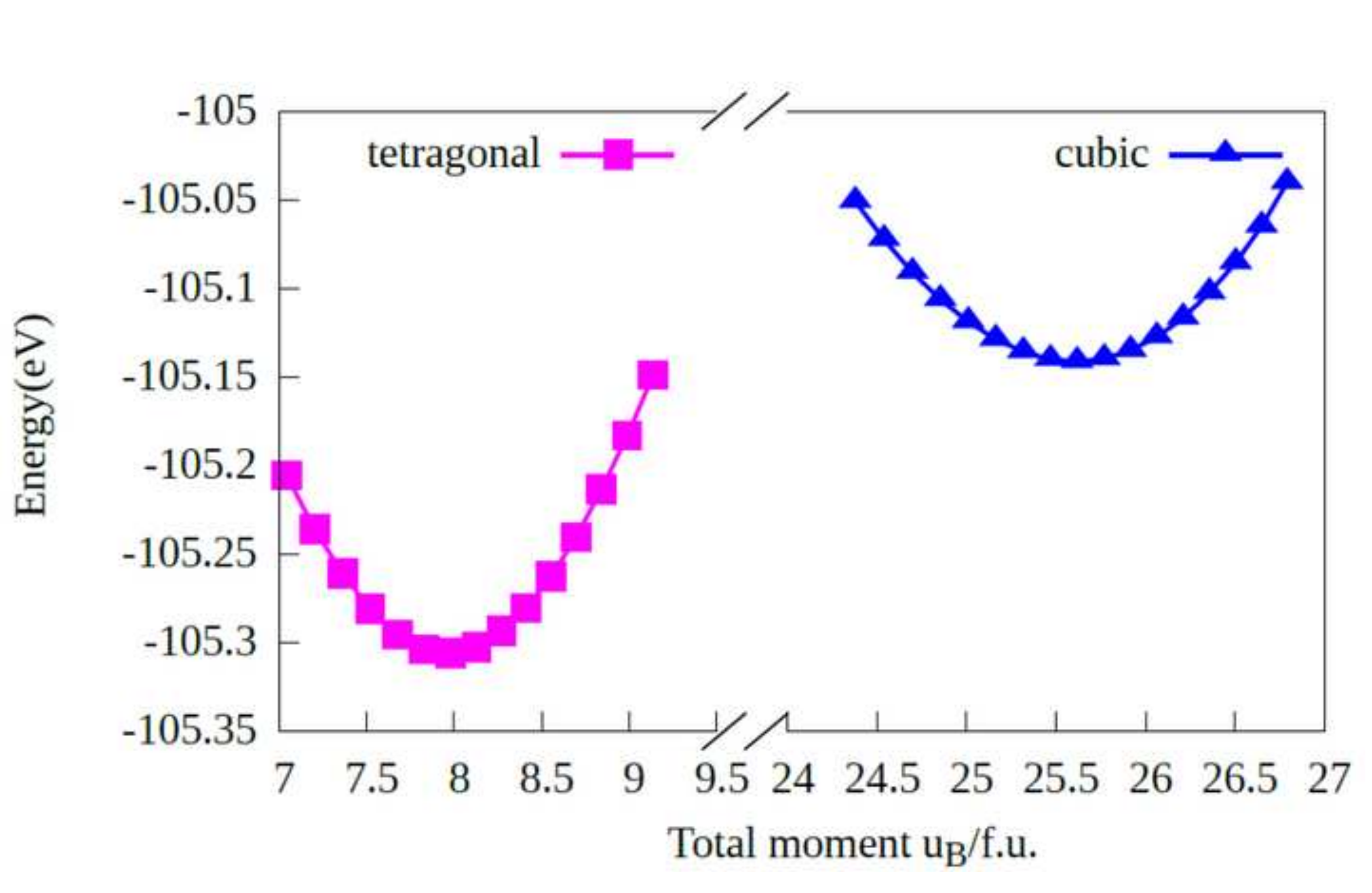




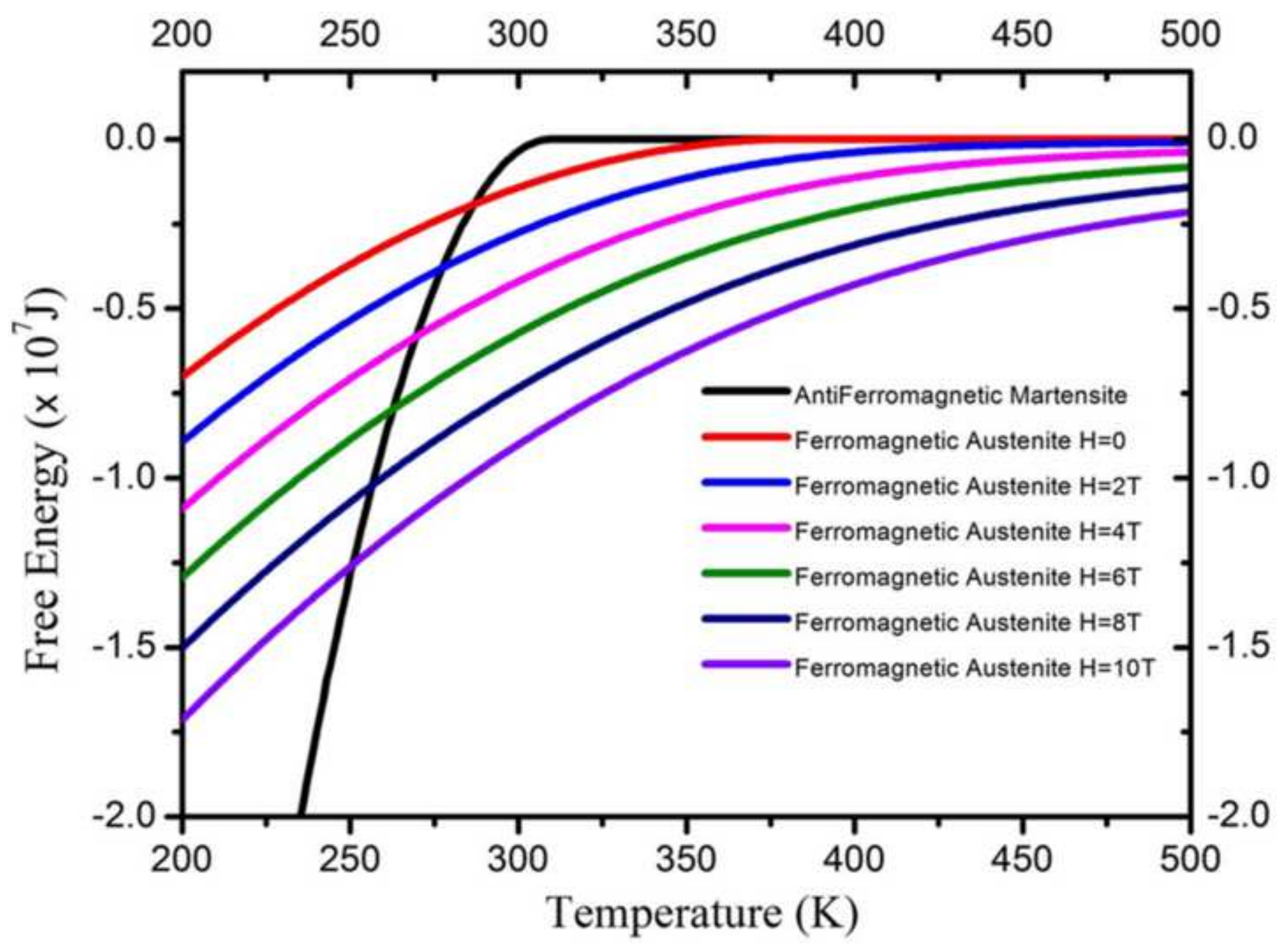




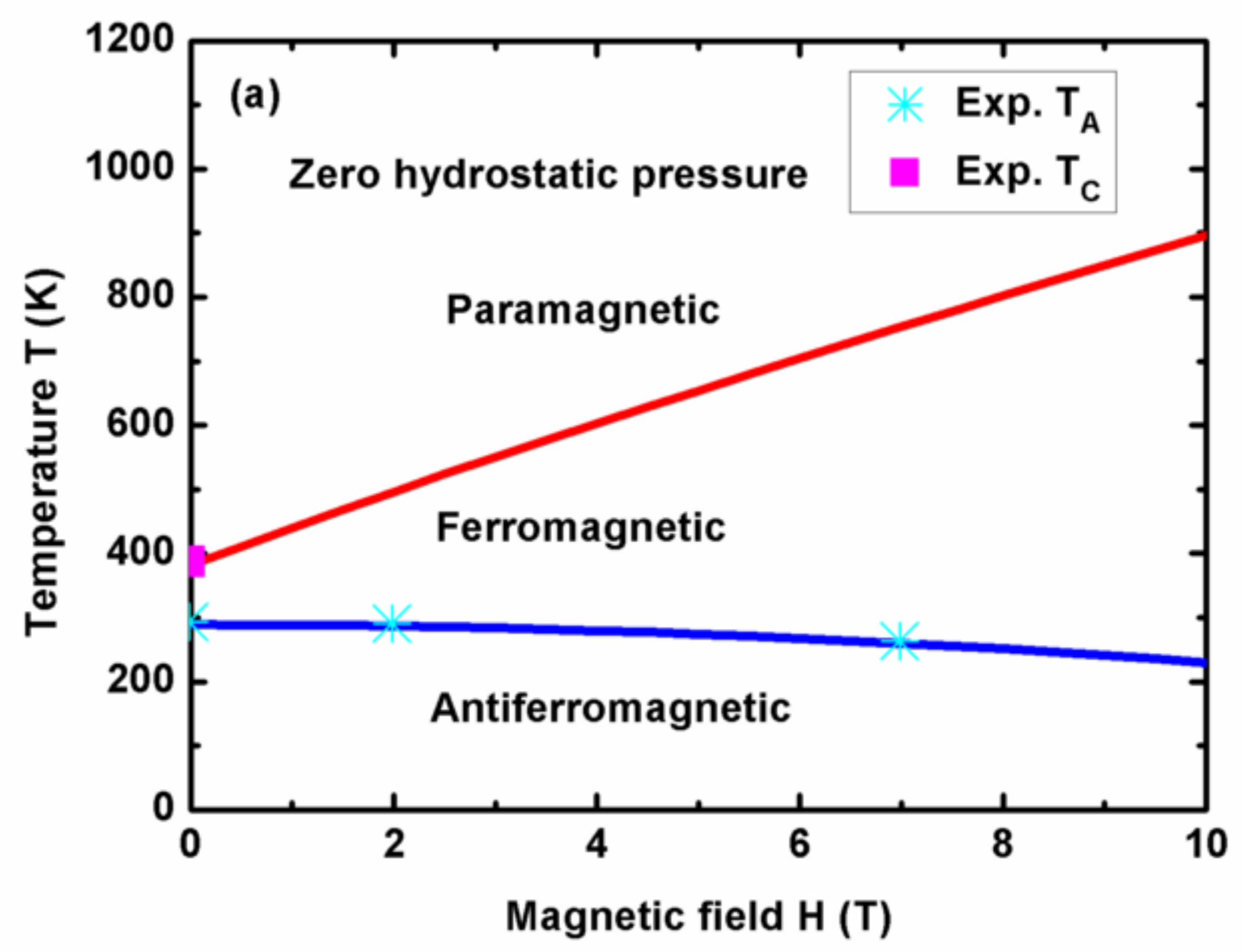




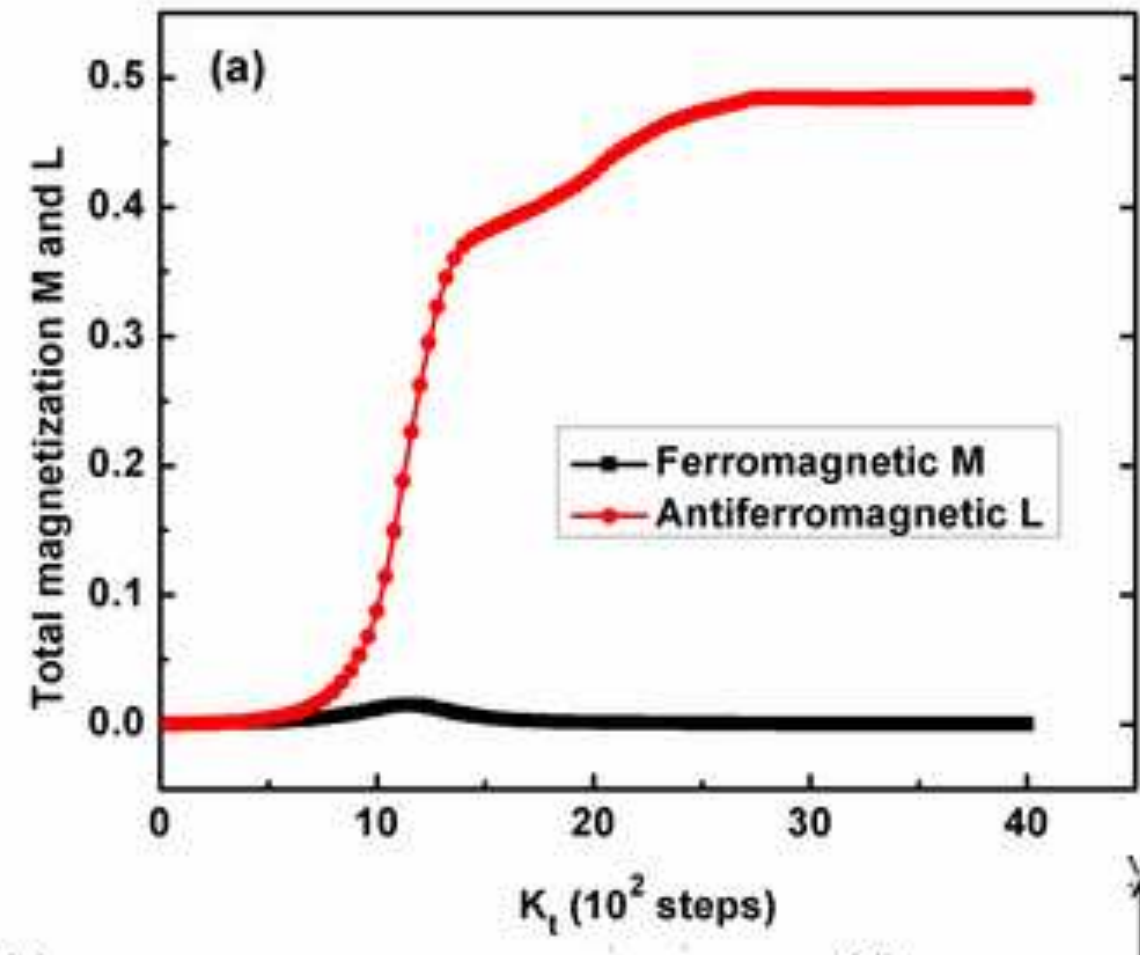

(c)

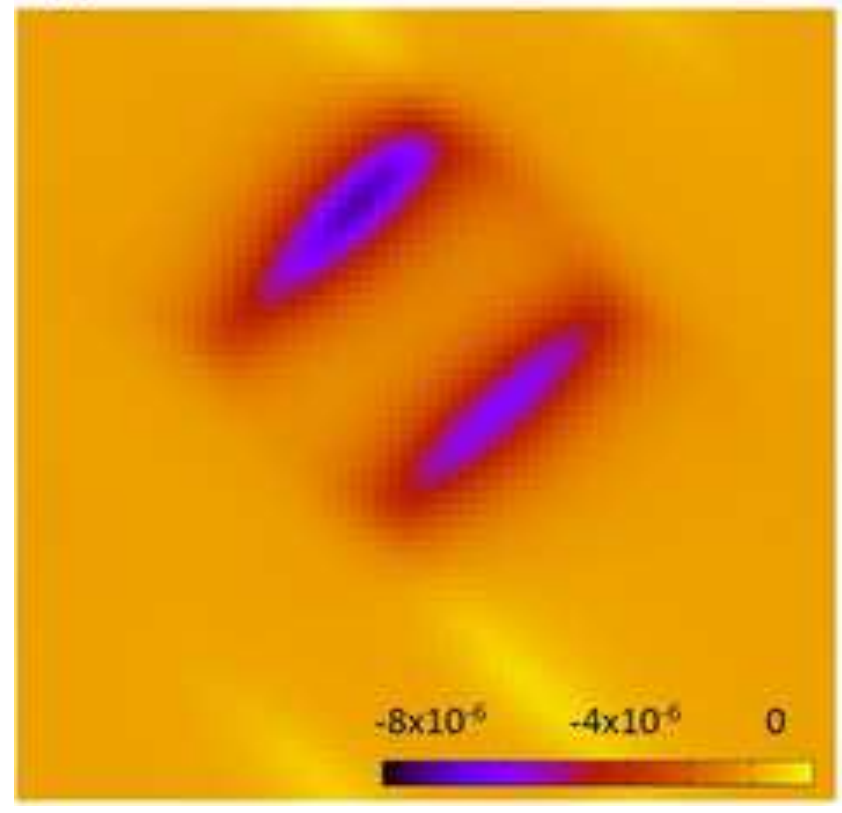

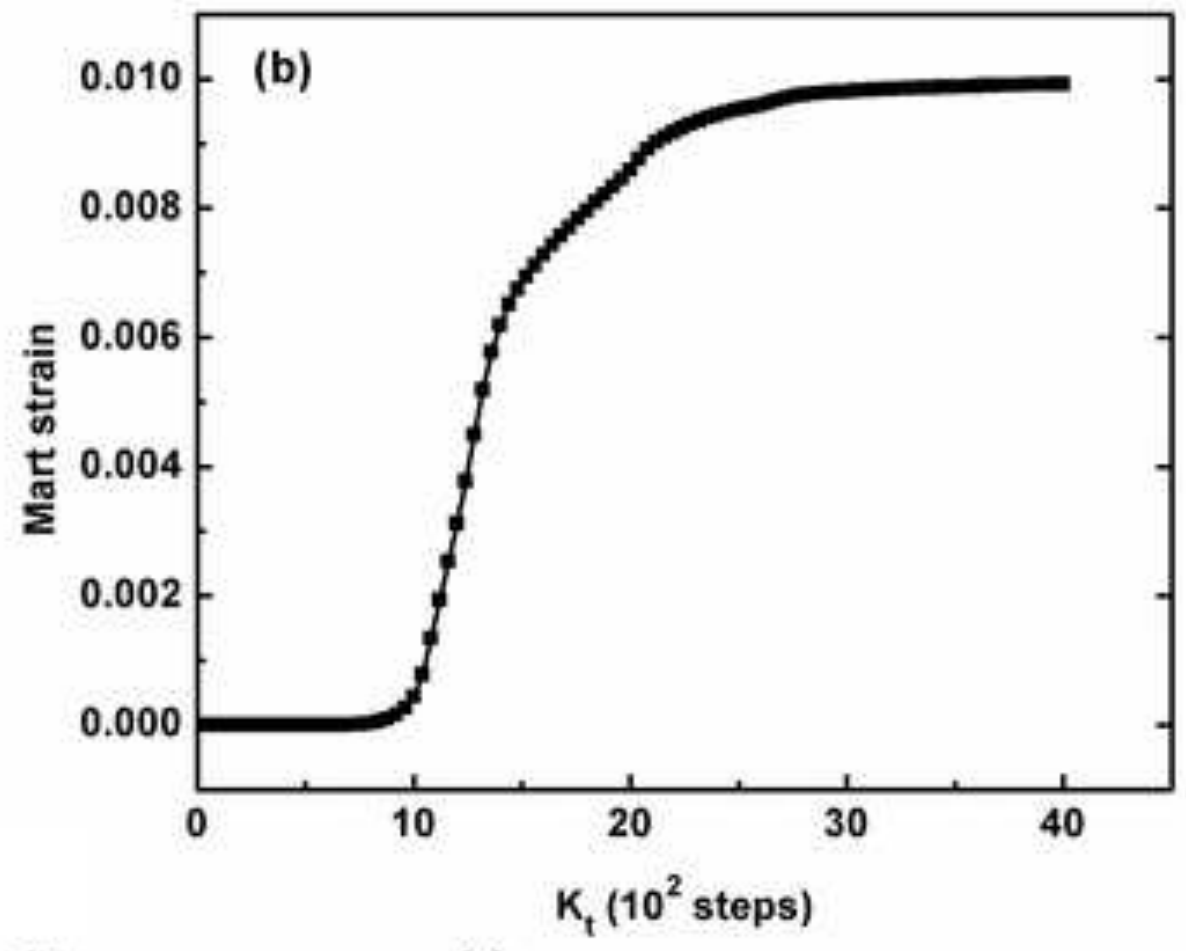

(e)
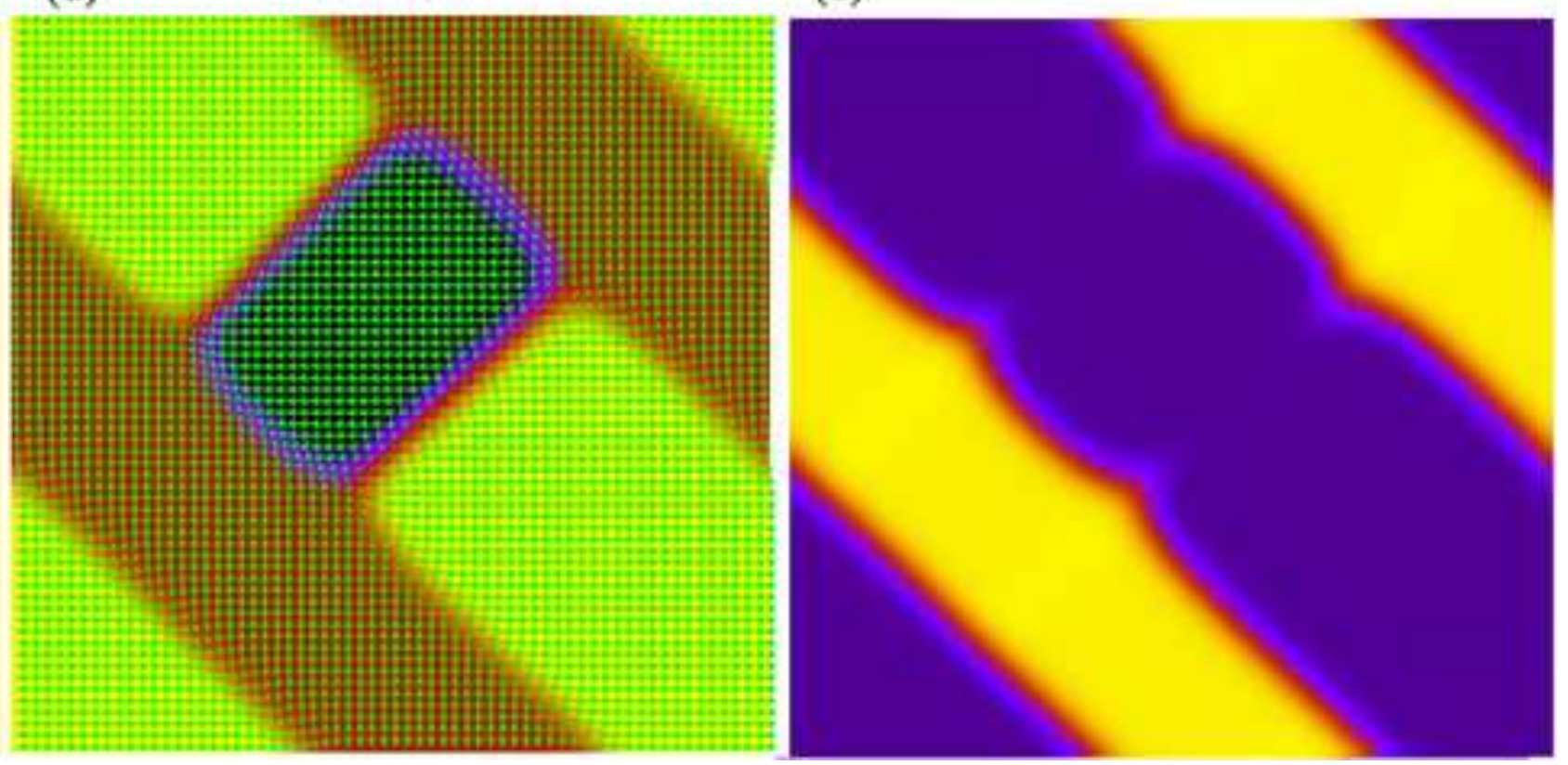


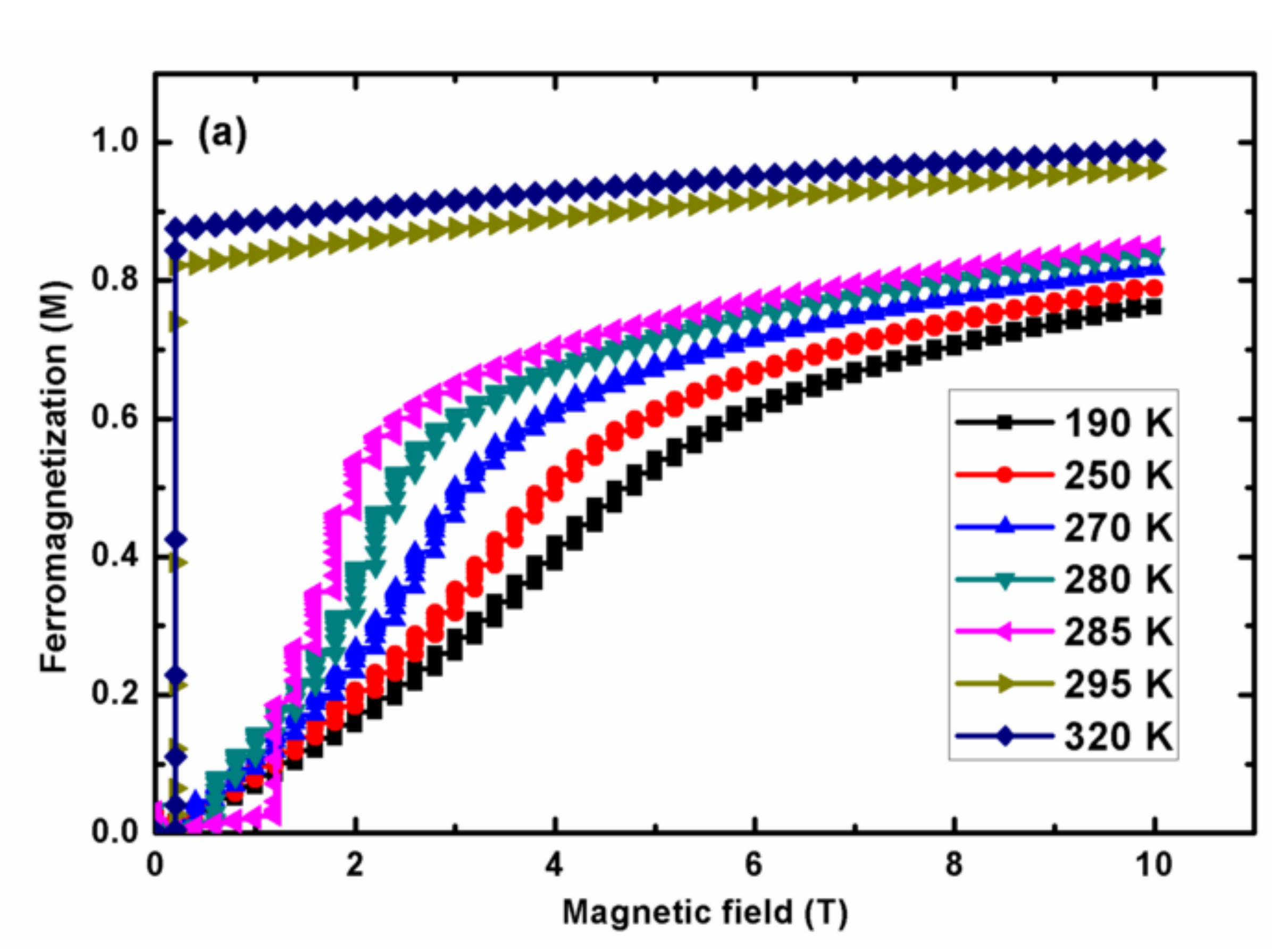




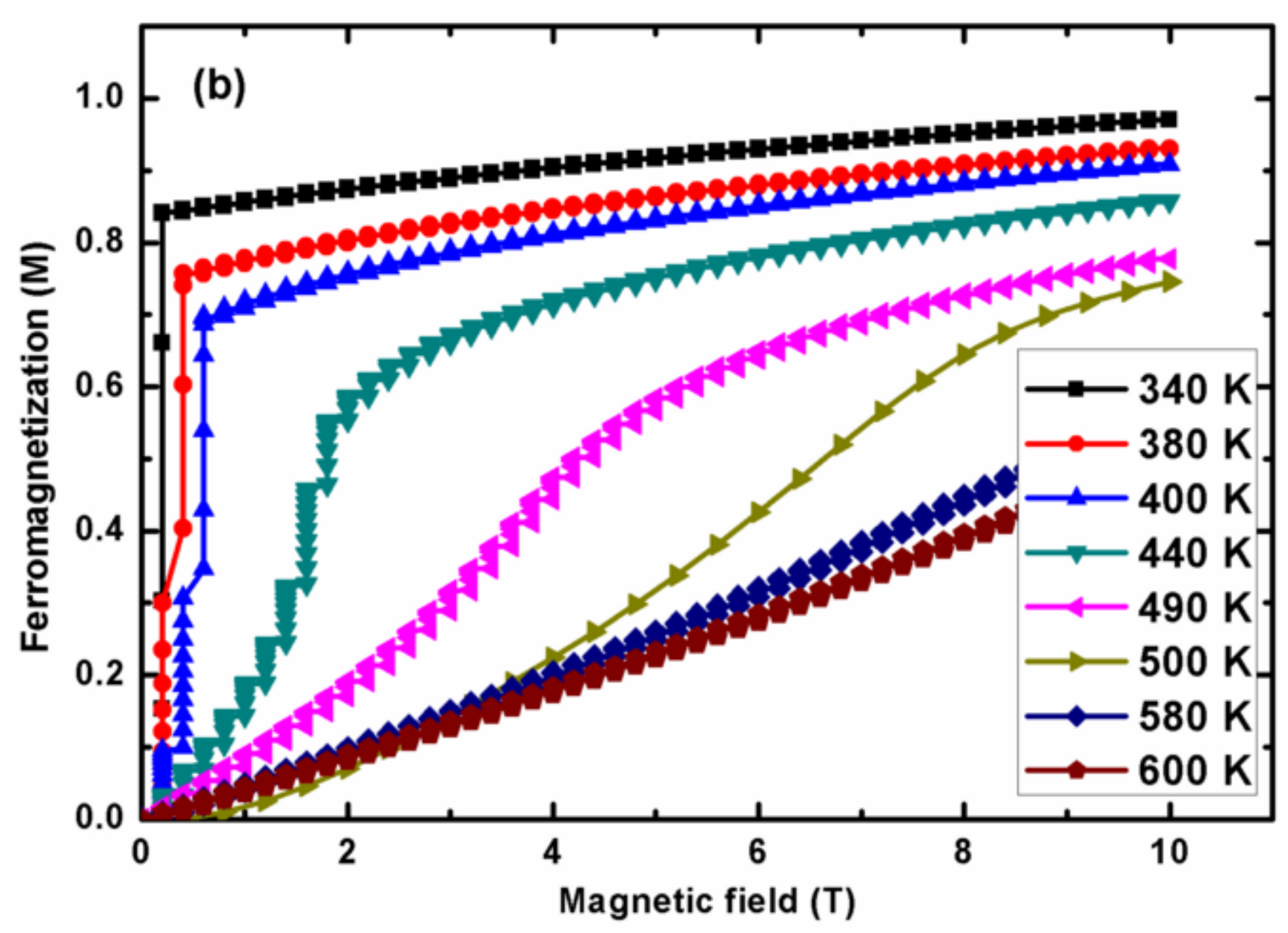

